\title{
Activity and Life After Survival of a Cardiac Arrest (ALASCA) and the effectiveness of an early intervention service: design of a randomised controlled trial
}

Véronique RMP Moulaert*1, Jeanine A Verbunt1,2, Caroline M van Heugten $^{3,4}$, Wilbert GM Bakx ${ }^{1}$, Anton PM Gorgels ${ }^{5}$, Sebastiaan CAM Bekkers ${ }^{5}$, Marc CFTM de Krom ${ }^{6}$ and Derick T Wade ${ }^{7,8}$

Address: ${ }^{1}$ Rehabilitation Foundation Limburg, Hoensbroek, The Netherlands, ${ }^{2}$ Department of General Practice, Maastricht University, Maastricht, The Netherlands, ${ }^{3}$ Department Brain and Behavior, Maastricht University, Maastricht, The Netherlands, ${ }^{4}$ Utrecht Centre of Excellence for Rehabilitation Medicine de Hoogstraat, Utrecht, The Netherlands, ${ }^{5}$ Department of Cardiology, University Hospital Maastricht, Maastricht, The Netherlands, ${ }^{6}$ Department of Neurology, University Hospital Maastricht, Maastricht, The Netherlands, ${ }^{7}$ Oxford Centre of Enablement, Oxford, UK and ${ }^{8}$ Care And Public Health Research Institute, Maastricht University, Maastricht, The Netherlands

Email: Véronique RMP Moulaert* - v.moulaert@srl.nl; Jeanine A Verbunt - j.verbunt@srl.nl; Caroline M van Heugten - c.vanheugten@np.unimaas.nl; Wilbert GM Bakx - w.bakx@srl.nl; Anton PM Gorgels - a.gorgels@cardio.azm.nl; Sebastiaan CAM Bekkers - b.bekkers@cardio.azm.nl; Marc CFTM de Krom - m.dekrom@neurologie.azm.nl; Derick T Wade - derick.wade@dsl.pipex.com

* Corresponding author

Published: 27 August 2007

BMC Cardiovascular Disorders 2007, 7:26 doi:10.1/86/147|-2261-7-26
Received: 17 July 2007

Accepted: 27 August 2007

This article is available from: http://www.biomedcentral.com/I47I-226I/7/26

(C) 2007 Moulaert et al; licensee BioMed Central Ltd.

This is an Open Access article distributed under the terms of the Creative Commons Attribution License (http://creativecommons.org/licenses/by/2.0), which permits unrestricted use, distribution, and reproduction in any medium, provided the original work is properly cited.

\begin{abstract}
Background: Cardiac arrest survivors may experience hypoxic brain injury that results in cognitive impairments which frequently remain unrecognised. This may lead to limitations in daily activities and participation in society, a decreased quality of life for the patient, and a high strain for the caregiver. Publications about interventions directed at improving quality of life after survival of a cardiac arrest are scarce. Therefore, evidence about effective rehabilitation programmes for cardiac arrest survivors is urgently needed. This paper presents the design of the ALASCA (Activity and Life After Survival of a Cardiac Arrest) trial, a randomised, controlled clinical trial to evaluate the effects of a new early intervention service for survivors of a cardiac arrest and their caregivers.
\end{abstract}

Methods/design: The study population comprises all people who survive two weeks after a cardiac arrest and are admitted to one of the participating hospitals in the Southern part of the Netherlands. In a two-group randomised, controlled clinical trial, half of the participants will receive an early intervention service.

The early intervention service consists of several consultations with a specialised nurse for the patient and their caregiver during the first three months after the cardiac arrest. The intervention is directed at screening for cognitive problems, provision of informational, emotional and practical support, and stimulating self-management. If necessary, referral to specialised care can take place. Persons in the control group will receive the care as usual.

The primary outcome measures are the extent of participation in society and quality of life of the patient one year after a cardiac arrest. Secondary outcome measures are the level of cognitive, emotional and cardiovascular impairment and daily functioning of the patient, as well as the strain 
for and quality of life of the caregiver. Participants and their caregivers will be followed for twelve months after the cardiac arrest.

A process evaluation will be performed to gain insight into factors that might have contributed to the effectiveness of the intervention and to gather information about the feasibility of the programme. Furthermore, an economic evaluation will be carried out to determine the costeffectiveness and cost-utility of the intervention.

Discussion: The results of this study will provide evidence on the effectiveness of this early intervention service, as well as the cost-effectiveness and its feasibility.

Trial registration: Current Controlled Trials [ISRCTN74835019]

\section{Background}

The incidence of cardiac arrest is $1-2$ per 1000 inhabitants a year but differs according to country and region [1]. In the Netherlands, the incidence of cardiac arrests lies between 0.6 - 0.9 per 1000 inhabitants per year, resulting in approximately 16,000 cases of cardiac arrests each year $[2,3]$. Resuscitation is attempted in $30-50 \%$ of the cases [4]. The survival rate at discharge from the hospital after attempted resuscitations is only 9 to $16 \%$ for out-of-hospital resuscitations [2,5-7] and between 14 and 37\% for in-hospital resuscitations [8-11]. Faster access to external automated defibrillators (AEDs) outside the hospital will probably raise survival rates $[4,12]$. This will lead to an increased number of cardiac arrest survivors and, thus, to more patients having to live with the consequences of surviving a cardiac arrest.

A cardiac arrest may lead to irreversible brain damage, called posthypoxic encephalopathy or hypoxic brain injury. Hypoxic brain injury often leads to cognitive or emotional impairments, with memory disorders and depressive symptoms being the most common complaints [13-15]. As a result of this, the performance of daily activities can be limited even six to twelve months later, with almost a quarter of the people needing some form of assistance in daily life [13,16-19]. Furthermore, participation in society often decreases after a cardiac arrest. For example, of the people who were working prior to a cardiac arrest only $20 \%$ were able to resume their job $[20,21]$. This all seems to result in a reduced quality of life for both the patient and their caregiver and leads to a high strain on the carer $[18,20-22]$.

Unfortunately, although the number of problems is substantial, a large percentage of these problems, especially the cognitive impairments, are not recognised or treated.

Currently, there exist no specific follow-up programmes or nursing interventions for cardiac arrest survivors in the Netherlands. In the international literature, only a few programmes for cardiac arrest survivors were found. In a study by Cowan et al., psychosocial nursing therapy reduced two-year mortality in a group of cardiac arrest survivors [23]. This intervention consisted of physiological relaxation (biofeedback), cognitive behavioral therapy aimed at self-management and health education. Dougherthy et al. showed that an intervention, based on the principles of social cognitive theory and the concerns of patients and their partners, reduced physical symptoms and anxiety and enhanced knowledge in persons who had received an internal cardioverter defibrillator (ICD) implantation [24-26]. However, this intervention had no effect on health care use $[25,26]$.

The diffuse brain injury and cognitive loss after a cardiac arrest seems in many ways similar to that seen after traumatic brain injury. In a study on traumatic brain injury patients, a routine follow up service resulted in a reduction in social morbidity and severity of post concussion symptoms six months later [27]. Given that cognitive impairment is a major problem in both hypoxic brain injury and moderate head injury, a comparable type of treatment could prove beneficial for patients after a cardiac arrest.

\section{Aims and hypotheses}

The primary goal of the present study is to evaluate the effectiveness of a new, early intervention service for survivors of a cardiac arrest and their caregivers. Secondly, a process evaluation will be performed to gain insight into factors that might have contributed to the effectiveness of the intervention and to gather information about the feasibility of the programme. Finally, an economic evaluation will determine the cost-effectiveness and cost-utility of the intervention.

We anticipate that the prospective follow-up and support provided by a specialised nurse will reduce the negative consequences of a cardiac arrest and will result in a higher level of participation in society and quality of life of the cardiac arrest survivor. Furthermore, we expect that this intervention will be cost-effective. 


\section{Methods/Design \\ Study design}

The study presented in this paper is a randomised, controlled clinical trial in which the effect of an early intervention service will be investigated. Participants will be followed over one year. During this year, three measurements will take place, namely two weeks, three months and twelve months after the cardiac arrest (see flow diagram, Figure 1). The Medical Ethics Committee of University Hospital Maastricht/Maastricht University approved the study protocol. The study is registered in a public trial registry [ISRCTN74835019]. This study is part of a larger research project, which also comprises a prospective and prognostic cohort study.

\section{Setting}

Participants will be recruited from the coronary care units and intensive care units of four hospitals in the Southern part of the Netherlands, starting in April 2007. One of the participating hospitals is a university hospital.

\section{Study population}

The population of this study consists of cardiac arrest survivors admitted to one of the participating hospitals, including both survivors of out-of-hospital and in-hospital cardiac arrests (for in- and exclusion criteria, see Table 1) and their caregivers. In this study caregiver is defined as

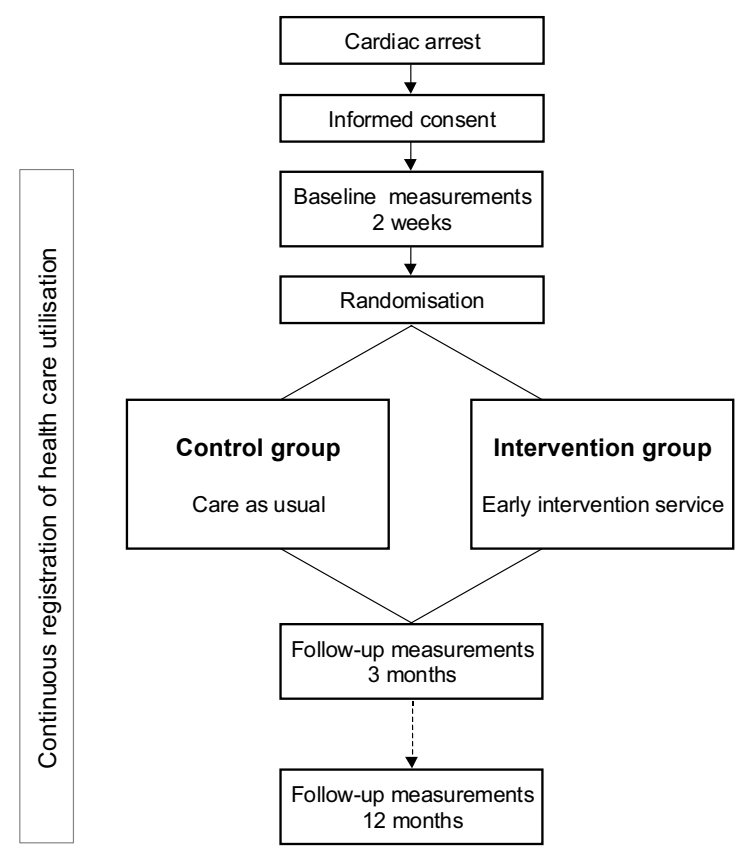

Figure I

Study design.
Table I: Inclusion and exclusion criteria

Inclusion criteria

- All people who survive two weeks after a cardiac arrest

- Admitted in or to one of the participating hospitals

- Living within $50 \mathrm{~km}$ of one of the participating hospitals

- 18 years or older

- Sufficient knowledge of Dutch language

Exclusion criteria

- Life expectancy lower than 3 months (as assessed by the treating physician)

- Living in residential or institutional care prior to the cardiac arrest

partner, spouse, or significant other that is most closely related to the patient.

The sample size calculation (two groups and one-sided testing) results from research on traumatic brain injured patients with regard to their participation in society assessed using the Community Integration Questionnaire (CIQ, described in more detail later). The mean (sd) total score on the CIQ was 16.09 ( $\mathrm{sd}=4.20)$ [28]. An assumed clinical relevant difference between groups of at least $10 \%$, an alpha of 0.05 and a power $(1-\beta)$ of 0.8 would necessitate 84 patients in each group. With an estimated loss to follow-up of $15 \%$, based on earlier research in cardiac arrest survivors, 200 participants are thought to be necessary for the clinical trial $[19,21,29]$. This should be achievable within three years, as the area served by the participating hospitals covers approximately 1,000,000 inhabitants.

\section{Recruitment and randomisation}

Between three and ten days after the cardiac arrest the patient and caregiver will be approached to participate in the study by their treating physician. Standard procedures concerning informed consent will be used. Both patients and caregivers sign an informed consent form if they decide to participate in the study. In case the patient is still unconscious or incapacitated at the moment that informed consent is needed, the caregiver will be asked to give provisional informed consent. When the patient regains consciousness, definitive informed consent will be sought.

Participants will be randomly allocated to either the intervention group or the control group. The randomisation will take place after the baseline measurements ( 2 weeks after the cardiac arrest) and will be performed centrally by the project leader using a computerised block randomisation. The randomisation scheme includes prestratification on two variables, namely hospital site and location of the cardiac arrest (in-hospital versus out-of hospital). Two series of opaque numbered envelopes will be prepared for each hospital site (one for in-hospital and one for out-ofhospital cardiac arrests), filled with a card indicating the 
allocated group. After registration, the envelope will be opened and the specialised nurse will be informed about the patients that are allocated to the intervention group.

\section{Group allocation and intervention}

The intervention group will receive an early intervention service delivered by a specialised nurse in addition to usual health care available to all patients, whereas the control group will only receive usual health care. The intervention period will extend up to three months after the cardiac arrest. During this period a specialised nurse will provide one to six consultations for the patient together with his or her caregiver (if present). The number of consultations will be tailored for each individual participant and will depend on the severity of the problems and the needs and wished of the patient and their caregiver. The consultations will take place in the hospital or at the participants' home. In between the consultations the participants have the opportunity to contact the nurses by phone or email. During the intervention period the specialised nurse will screen for cognitive and emotional problems. Next to that, information will be provided by means of an information booklet and by discussing questions that the participants may have. Furthermore, emotional and practical support will be offered and selfmanagement will be explained and promoted. A consultant in rehabilitation medicine supervises the specialised nurses. If necessary, referral to additional diagnostics or specialised rehabilitation care can take place.

There will be no restrictions on possible co-interventions. Participants assigned to the intervention group who do not want treatment will still be approached for all the follow-up measurements.

\section{Data collection}

Baseline measurements will be administered two weeks (= T1) after the cardiac arrest, with further assessments at three $(=\mathrm{T} 2)$ and twelve months $(=\mathrm{T} 3)$. The measurements will be administered wherever the patient is at that time. A research assistant, who will be blinded to the group allocation, will perform the assessments.

To check for the effectiveness of blinding, the research assistants will be asked after the last measurement to indicate whether they think the participant belonged to the intervention group or the control group.

\section{Socio-demographical and medical variables}

The following socio-demographical variables will be recorded: Age, gender, nationality, marital status, living condition, educational level, and work situation. The variables related to the resuscitation and medical situation of the patient that will be recorded are presented in Table 2 .
Table 2: Variables related to the resuscitation and the medical condition of the patient.

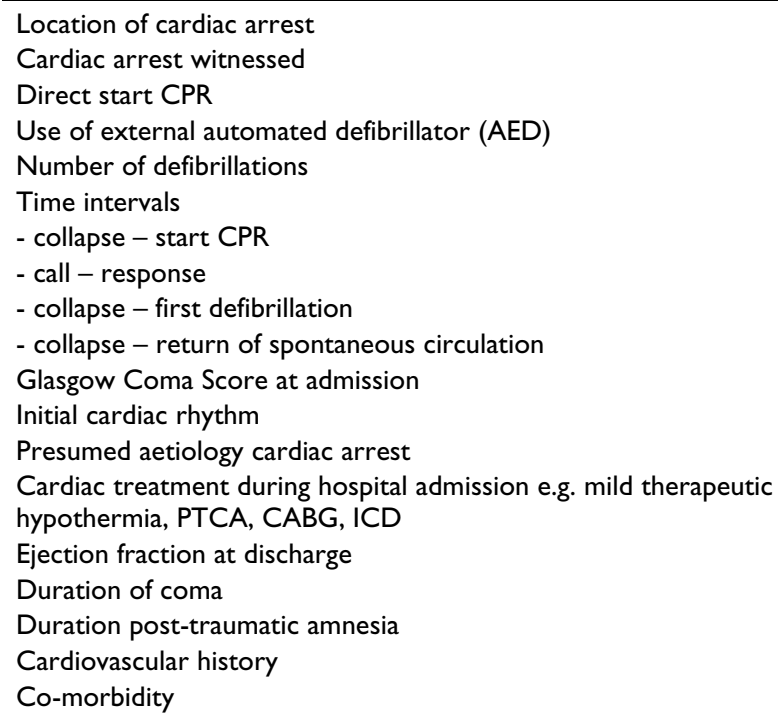

\section{Outcome measures for the effect evaluation}

The primary outcome measures of this trial are participation in society and quality of life of the cardiac arrest survivor. The secondary outcome measures are cognitive, emotional and cardiovascular impairment and daily functioning of the cardiac arrest survivor, as well as the strain, emotional functioning and quality of life of the caregiver. The instruments that will be used to measure these domains are presented in Table 3 and Table 4 and are described in the next sections.

\section{Primary outcome measures Community Integration Questionnaire}

The Community Integration Questionnaire (CIQ) is a 15item questionnaire that assesses the level of participation in society [30]. The CIQ comprises three scales, namely home integration, productive activity, and social integration.

\section{Impact on Participation and Autonomy}

The Impact on Participation and Autonomy (IPA) is a 39item questionnaire that focuses on two aspects of participation, namely perceived participation and the experience of problems [31].

\section{EuroQol 6D}

The EuroQol 6D (EQ-6D) is a generic quality of life measure consisting of 6 items (mobility, self-care, usual activities, pain/discomfort, anxiety/depression and cognition) each with three answer possibilities [32]. In addition, participants are asked to give a general rating of their current 
Table 3: Outcome domains, measurement instruments and measurement moments for the cardiac arrest survivors

\begin{tabular}{|c|c|c|c|c|c|c|}
\hline \multirow[b]{2}{*}{ Domain } & \multirow[b]{2}{*}{ Measurement instrument } & \multirow[b]{2}{*}{ Abbr. } & \multicolumn{4}{|c|}{ Measurement moments } \\
\hline & & & T0 & TI & T2 & T3 \\
\hline \multirow[t]{2}{*}{ Participation in society } & Community Integration Questionnaire & ClQ & $x$ & & $x$ & $\mathrm{X}$ \\
\hline & Impact on Participation and Autonomy & IPA & & & & $x$ \\
\hline \multirow{3}{*}{ Quality of life } & Short-Form-36 (RAND 36-item Health Survey) & SF-36 & & $\mathrm{x}$ & $\mathrm{x}$ & $\mathrm{X}$ \\
\hline & EuroQol 6D & EQ-6D & & $x$ & $x$ & $x$ \\
\hline & Quality Of Life after Brain Injury & QOLIBRI & & & $x$ & $\mathrm{X}$ \\
\hline \multirow[t]{6}{*}{ Cognitive functioning } & Cognitive Failures Questionnaire & CFQ & & $x$ & $x$ & $x$ \\
\hline & Cognitive Log & Cog-log & & $x$ & $x$ & $\mathrm{X}$ \\
\hline & Adult Memory and Information Processing Battery & AMIPB & & $x$ & $x$ & $x$ \\
\hline & Paragraph Recall & PR & & $\mathrm{x}$ & $\mathrm{x}$ & $x$ \\
\hline & Trail Making Test, part A + B & TMT & & $x$ & $x$ & $\mathrm{X}$ \\
\hline & Verbal Fluency Test & VFT & & $x$ & $x$ & $\mathrm{X}$ \\
\hline \multirow[t]{2}{*}{ Emotional functioning } & Hospital Anxiety and Depression Scale & HADS & & $x$ & $x$ & $x$ \\
\hline & Impact of Event Scale & IES & & $x$ & $x$ & $x$ \\
\hline Cardiorespiratory functioning & New York Heart Association Classification & NYHA & & $x$ & $x$ & $\mathrm{X}$ \\
\hline Fatigue & Fatigue Severity Scale & FSS & & & $x$ & $x$ \\
\hline Basic daily activities & Barthel Index & $\mathrm{BI}$ & & $x$ & & \\
\hline Instrumental daily activities & Frenchay Activity Index & FAI & $x$ & & $x$ & $x$ \\
\hline
\end{tabular}

T0 = situation prior to cardiac arrest (asked retrospectively at $\mathrm{TI}$ )

$\mathrm{TI}=2$ weeks after cardiac arrest

$\mathrm{T} 2=3$ months after cardiac arrest

$\mathrm{T} 3=1$ year after cardiac arrest

health state on a visual analogue scale, ranging from 0 to 100.

Medical Outcomes Study 36-item Short Form Health Survey The Medical Outcomes Study 36-item Short Form Health Survey (SF-36/RAND 36-item Health Survey) is a 36-item generic quality of life questionnaire that measures several domains of perceived health [33].

Quality Of Life after Brain Injury

The Quality Of Life after Brain Injury questionnaire (QOLIBRI) is a brain injury specific quality of life measure. The QOLIBRI consists of 49 questions that measure health related quality of life within six domains (physical condition, thinking activities, feelings and emotions, functioning in daily life, relationships and social/leisure activities, current situation and future prospects) [34].

\section{Secondary outcome measures}

Cognitive Failures Questionnaire

The Cognitive Failures Questionnaire (CFQ) is a 25-item questionnaire on self-reported cognitive failures [35]. Both the patient as well as his or her caregiver will be asked to fill out this questionnaire to evaluate the cognitive status of the patient.

\section{Cognitive Log}

The Cognitive Log (Cog-log) is a 10-item cognitive screening instrument [36]. It measures higher neurocognitive

Table 4: Outcome domains, measurement instruments and measurement moments for the caregivers

\begin{tabular}{|c|c|c|c|c|c|}
\hline \multirow[b]{2}{*}{ Domain } & \multirow[b]{2}{*}{ Measurement instrument } & \multirow[b]{2}{*}{ Abbr. } & \multicolumn{3}{|c|}{ Measurement moments } \\
\hline & & & TI & T2 & T3 \\
\hline \multirow[t]{2}{*}{ Quality of life } & Short-Form-36 (RAND 36-item Health Survey) & SF-36 & $X$ & $\mathrm{X}$ & X \\
\hline & EuroQol 6D & EQ-6D & $X$ & $X$ & $\mathrm{X}$ \\
\hline \multirow[t]{2}{*}{ Emotional functioning } & Hospital Anxiety and Depression Scale & HADS & $X$ & $X$ & $X$ \\
\hline & Impact of Event Scale & IES & $X$ & $X$ & $x$ \\
\hline Fatigue & Fatigue Severity Scale & FSS & & $X$ & $\mathrm{X}$ \\
\hline Caregiver Strain & Caregiver Strain Index & CSI & $X$ & $x$ & 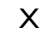 \\
\hline
\end{tabular}

$\mathrm{TI}=2$ weeks after cardiac arrest

$\mathrm{T} 2=3$ months after cardiac arrest

$\mathrm{T} 3=1$ year after cardiac arrest 
processes including orientation, memory, concentration and executive skills.

\section{Adult Memory and Information Processing Battery Task A}

The Adult Memory and Information Processing Battery (AMIPB) Task A is a measure for information processing speed [37]. In this test, the subject is asked to select the second highest number in each row of five. The final score is the number of correct choices made in two minutes.

\section{Paragraph Recall}

The Paragraph Recall (PR) is a test for immediate and delayed verbal memory [38]. The subject is asked to recall as much as possible from a paragraph, which is read to the subject aloud. This will be performed both directly, and, without warning, again 20 minutes later. To prevent interference with the paragraph from the previous measurement, a different paragraph will be used each time.

\section{Trail Making Test}

The Trail Making Test (TMT) measures scanning, visuomotor tracking, divided attention and cognitive flexibility [39]. The test is divided into two parts, part A and B. In part $\mathrm{A}$, the subject is asked to connect consecutively numbered circles on one work sheet as fast as possible. In part $\mathrm{B}$, the subject is asked to connect consequently numbered and lettered circles. The time needed to complete part A and part $\mathrm{B}$ is recorded.

\section{Verbal Fluency Test}

During the Verbal Fluency Test (VFT), the subject is asked to name as many words from one semantic category, in this case animals, in one minute [40].

\section{Hospital Anxiety and Depression Scale}

The Hospital Anxiety and Depression Scale (HADS) is a commonly used questionnaire, which was designed to detect the presence of mild degrees of mood disorders in non-psychiatric hospital outpatients [41]. The HADS consists of 14 items, and has two sub-scales, namely depression and anxiety.

\section{Impact of Event Scale}

The Impact of Event Scale (IES) measures psychological reactions that can take place after a traumatic event. The IES has 15 items and explores the level of posttraumatic stress by asking for intrusive and avoidance symptoms $[42,43]$.

\section{New York Heart Association Classification}

The New York Heart Association Classification (NYHAclassification) is a functional classification system that divides cardiac patients into four classes depending on their limitation in physical activities [44].

\section{Fatigue Severity Scale}

The Fatigue Severity Scale (FSS) is a 9-item questionnaire that measures experienced severity of fatigue symptoms in daily activities [45].

\section{Barthel Index}

The Barthel Index (BI) is a widely used instrument that measures basic (personal) activities of daily living (ADL). The instrument consists of 10 items and measures to what extent a person can perform basic ADL activities independently [46].

\section{Frenchay Activities Index}

The Frenchay Activities Index (FAI) is an accepted measure for instrumental ADL that reports on fifteen daily life activities [47]. Compared to the Barthel Index, the measured activities are of a higher level, for example the preparation of meals, doing groceries, making trips and performing paid labour.

\section{Caregiver Strain Index}

The Caregiver Strain Index (CSI) is a 13-item, dichotomous scale that has to be filled out by a partner, caregiver or significant other [48]. The CSI measures strain related to the provision of care.

\section{Parameters for process evaluation}

The intervention will be evaluated based on information from the specialised nurses and the participants. First, the intervention process will be evaluated based on information provided by the specialised nurse. He/she will record the following items: the actual number of consultations (face-to-face, by telephone and by email), the direct time related to each consultation, the indirect time related to each consultation (administration, travel time), the provision of information per consultation, the use of the screening tools, the kind of problems presented, number of referrals, completion of the intervention according to protocol and, in case of non-compliance, reason for noncompliance. Second, after completion of the intervention the participant and his/her caregiver will be asked to fill out a questionnaire to evaluate the intervention.

\section{Determination of costs}

Data concerning costs will be gathered prospectively using monthly cost-diaries [49]. All the participants receive costdiaries in which they are asked to note all their health care utilisation during that month. To prevent non-compliance, the research assistant will give a phone call at the end of each month and will record the health care utilisation of that month. For the calculation of the total costs, three categories can be distinguished, namely direct health care costs, direct non-health care costs and indirect costs. For the determination of the direct health care costs the following variables will be recorded: duration admis- 
sion in health care facility, visits physicians and other health care providers and amount of prescribed medication. Direct non-health care costs that will be measured, are the costs of informal health care, over-the-counter medication, costs of health activities, hours of paid or unpaid household help and special aids. The indirect costs comprise loss of paid and unpaid work productivity of the patient.

\section{Statistical analysis}

Effect evaluation

Descriptive techniques will be used to present data concerning the participants, number of dropouts, losses-tofollow up and the scores on the outcome measures.

To determine whether the patients participating in this trial are representative for the population of cardiac arrest survivors, the baseline characteristics between compliant and non-compliant participants, as well as dropouts and losses-to-follow up will be compared.

Before examining the effectiveness of the intervention, the comparability of the two groups will be checked. Baseline characteristics will be compared using independent sample T-test (normal distribution) or Mann Whitney U-test (non-normal distribution), in case of a continuous variable. In case of a dichotomous variable a chi-square test will be used.

The data from the evaluation study will be analysed according to the intention-to-treatment principle. If either $\mathrm{T} 1$ or $\mathrm{T} 2$ data are missing the 'last-observation-carried-forward' principle will be used.

Multiple regression analysis, adjusted for possible differences in baseline characteristics or baseline scores, will be applied to examine differences between the intervention and the control group on the primary outcome measures at T3 (one year after the cardiac arrest). If there is a difference between the groups, the effect size will be calculated. Subgroup analysis will be performed for potential effect modifiers.

To assess whether protocol deviations or care provided outside the intervention have caused biases, the results of the intention-to-treatment analysis will be compared to the on-treatment analysis.

Process evaluation

The outcomes of the process evaluation will be reported using descriptive techniques.

\section{Economic evaluation}

To determine the cost-effectiveness of the intervention a statistical analysis of costs will be performed. The total costs are calculated by adding up direct health care costs, direct non-health care costs and indirect costs. Health care costs are estimated using the Dutch guideline for cost analysis in health care research [50]. The Friction Cost Approach will be used to evaluate production losses, necessary to determine indirect costs [50]. Discounting of costs will take place if significant changes in health care costs take place during the study period. As the distribution of costs might be skewed, differences in costs between the intervention and care-as-usual will be calculated by means of bootstrapping, a method appropriate for any distribution of data.

A multi-way sensitivity analysis will be performed to gain insight into the generalisability of the economic evaluation.

A cost-utility analysis will relate the difference in between the intervention and care-as-usual to changes in utility. This will result in costs per quality-adjusted-life-years (QALY's).

\section{Discussion}

The main goal of the ALASCA trial is to evaluate a new early intervention service for cardiac arrest survivors and their caregivers. This is a relevant topic because evidence on effective rehabilitation programmes for cardiac arrest survivors is urgently needed. During the design of this study many choices and selections were made, and four of the more important ones will be discussed.

First, the intervention was designed especially for this study. There are very few studies describing programmes for people with hypoxic brain injury due to a cardiac arrest. Consequently, we chose to model the intervention upon evidence of effective interventions for people who had traumatic brain injury, as there are many similarities between hypoxic and traumatic brain injuries [27]. This intervention designed for this study is a combination of a psychosocial intervention and a process intervention. The psychosocial aspects of the intervention are directed at providing informational, emotional and practical support and simulating self-management. The intervention is also process intervention because it is directed at providing the care needed fast and efficiently to each individual patient.

Second, the selection of our primary outcome measures, participation in society and quality of life, may differ from common primary outcome measures in cardiology research. However, this is a common approach in rehabilitation medicine, where maximisation of participation in society is one of the main goals [51]. We expect that through the care provided in the new intervention, patients will be able to reach a higher level of participation, as well as a higher quality of life. 
Third, it may need some clarification why three quality of life measures will be administered. The EQ-6D and the SF36 were chosen because they measure generic quality of life and can be used in the cost-effectiveness analyses. Next to that, these questionnaires have been used in many other patient groups, which makes it possible to compare the quality of life of cardiac arrest survivors to that of other patient groups. The third quality of life measure is the QOLIBRI, which is a brain injury specific questionnaire. This questionnaire was included because it may have a higher sensitivity to change in case of hypoxic brain injury than both general quality of life measures.

Fourth, we explicitly chose to include the strain for and quality of life of the caregiver in the secondary outcome measures. Our impression is that being the partner of a cardiac arrest survivor can imply a heavy burden. These caregivers are at risk for developing stress and emotional problems and this definitely needs more attention. Therefore, the intervention is directed at both the patient and their caregiver, who is expected to benefit from the early intervention service as well.

To conclude, this paper describes the design of a randomised, controlled clinical trial that will investigate the effectiveness of a new early intervention service for cardiac arrest survivors and their caregivers. The inclusion of the participants started in April 2007 and will continue until April 2010. The results of this study will provide evidence on the effectiveness of this early intervention service, as well as the cost-effectiveness and its feasibility. This will give insight into the question whether it would be useful to implement this early intervention service in the Dutch health care system.

\section{Competing interests}

The author(s) declare that they have no competing interests.

\section{Authors' contributions}

VRMP is the main researcher and is responsible for writing the protocol. JAV and WGMB originated the idea for the study. JAV is project-leader of the study. JAV and $\mathrm{CMvH}$ are the supervisors of VRMP. DTW is the promotor of VRMP.

JAV, CMvH, WGMB, APMG, SCAMB, MCFTMdK and DTW participated in the design of the study and research protocol. All authors read and corrected draft versions of the manuscript and approved the final manuscript.

\section{Acknowledgements}

The study is funded by the Netherlands Organisation for Health Research and Development (ZON/MW) and Stichting Nuts Ohra.
We would like to thank Brigitte Cobben and Peter Heuts for their contribution to the design of the intervention. Jolanda van Haastregt gave useful advice about the process evaluation. Marielle Goossens is thanked for her help with regard to the design of the economic evaluation of this study.

\section{References}

I. Myerburg RJ, Castellanos A: Cardiac arrest and sudden cardiac death. In Heart Disease: A Textbook of Cardiovascular Medicine Sixth edition edition. Edited by: Braunwald E, Zipes DP and Libby P. Philadelphia, WB Saunders; 2001:890-93I.

2. Waalewijn RA, de Vos R, Koster RW: Out-of-hospital cardiac arrests in Amsterdam and its surrounding areas: results from the Amsterdam resuscitation study (ARREST) in 'Utstein' style. Resuscitation 1998, 38:157-167.

3. Gorgels AP, Gijsbers C, de Vreede-Swagemakers J, Lousberg A, Wellens HJ: Out-of-hospital cardiac arrest--the relevance of heart failure. The Maastricht Circulatory Arrest Registry. Eur Heart J 2003, 24: I 204-I 209.

4. Berdowski RA, Waalewijn RA, Koster RW: Overleving na reanimatie buiten het ziekenhuis is sterk toegenomen: een vergelijkend onderzoek tussen eind 20 ste en begin 2 Iste eeuw. In Hart- en vaatziekten in Nederland najaar 2006, cijfers over ziekte en sterfte Edited by: Vaartjes I, Peters RJG, van Dis SJ and Bots ML. Den Haag, Nederlandse Hartstichting; 2006.

5. Bottiger BW, Grabner C, Bauer H, Bode C, Weber T, Motsch J, Martin E: Long term outcome after out-of-hospital cardiac arrest with physician staffed emergency medical services: the Utstein style applied to a midsized urban/suburban area. Heart 1999, 82:674-679.

6. Kuisma M, Maatta T: Out-of-hospital cardiac arrests in Helsinki: Utstein style reporting. Heart 1996, 76:18-23.

7. Fischer M, Fischer NJ, Schuttler J: One-year survival after out-ofhospital cardiac arrest in Bonn city: outcome report according to the 'Utstein style'. Resuscitation 1997, 33:233-243.

8. Hodgetts TJ, Kenward G, Vlackonikolis I, Payne S, Castle N, Crouch $\mathrm{R}$, Ineson N, Shaikh L: Incidence, location and reasons for avoidable in-hospital cardiac arrest in a district general hospital. Resuscitation 2002, 54: I I5-I 23.

9. Huang $\mathrm{CH}$, Chen WJ, Ma MH, Chang WT, Lai CL, Lee YT: Factors influencing the outcomes after in-hospital resuscitation in Taiwan. Resuscitation 2002, 53:265-270.

10. Patrick A, Rankin N: The in-hospital Utstein style: use in reporting outcome from cardiac arrest in Middlemore Hospital 1995-1996. Resuscitation 1998, 36:91-94.

II. Skogvoll E, Isern E, Sangolt GK, Gisvold SE: In-hospital cardiopulmonary resuscitation. 5 years' incidence and survival according to the Utstein template. Acta Anaesthesiol Scand 1999. 43:177-184.

12. Valenzuela TD, Roe DJ, Nichol G, Clark LL, Spaite DW, Hardman RG: Outcomes of rapid defibrillation by security officers after cardiac arrest in casinos. N Engl J Med 2000, 343: I 206-I 209.

13. Sauve MJ, Doolittle N, Walker JA, Paul SM, Scheinman MM: Factors associated with cognitive recovery after cardiopulmonary resuscitation. Am J Crit Care 1996, 5:127-139.

14. Roine RO, Kajaste S, Kaste M: Neuropsychological sequelae of cardiac arrest. Jama 1993, 269:237-242.

15. Grubb NR, O'Carroll R, Cobbe SM, Sirel J, Fox KA: Chronic memory impairment after cardiac arrest outside hospital. $B m j$ 1996, 313:143-146.

16. Fertl E, Vass K, Sterz F, Gabriel H, Auff E: Neurological rehabilitation of severely disabled cardiac arrest survivors. Part I. Course of post-acute inpatient treatment. Resuscitation 2000, 47:231-239.

17. Lim C, Alexander MP, LaFleche G, Schnyer DM, Verfaellie M: The neurological and cognitive sequelae of cardiac arrest. Neurology 2004, 63: 1774-1778.

18. Pusswald G, Fertl E, Faltl M, Auff E: Neurological rehabilitation of severely disabled cardiac arrest survivors. Part II. Life situation of patients and families after treatment. Resuscitation 2000, 47:24I-248.

19. van Alem AP, de Vos R, Schmand B, Koster RW: Cognitive impairment in survivors of out-of-hospital cardiac arrest. Am Heart J 2004, | 48:4|6-42|. 
20. de Vos R, de Haes HC, Koster RW, de Haan RJ: Quality of survival after cardiopulmonary resuscitation. Arch Intern Med 1999, I 59:249-254.

21. Lundgren-Nilsson A, Rosen H, Hofgren C, Sunnerhagen KS: The first year after successful cardiac resuscitation: function, activity, participation and quality of life. Resuscitation 2005, 66:285-289.

22. Middelkamp W, Moulaert VR, Verbunt JA, van Heugten CM, Bakx WG, Wade DT: Life after survival: long-term daily life functioning and quality of life of patients with hypoxic brain injury as a result of a cardiac arrest. Clin Rehabil 2007, 2 I:425-43I.

23. Cowan MJ, Pike KC, Budzynski HK: Psychosocial nursing therapy following sudden cardiac arrest: impact on two-year survival. Nurs Res 200I, 50:68-76.

24. Dougherty CM, Johnson-Crowley NR, Lewis FM, Thompson EA Theoretical development of nursing interventions for sudden cardiac arrest survivors using social cognitive theory. ANS Adv Nurs Sci 200I, 24:78-86.

25. Dougherty CM, Lewis FM, Thompson EA, Baer JD, Kim W: Shortterm efficacy of a telephone intervention by expert nurses after an implantable cardioverter defibrillator. Pacing Clin Electrophysiol 2004, 27:1594-1602.

26. Dougherty CM, Thompson EA, Lewis FM: Long-term outcomes of a telephone intervention after an ICD. Pacing Clin Electrophysiol 2005, 28: II57-II67.

27. Wade DT, King NS, Wenden FJ, Crawford S, Caldwell FE: Routine follow up after head injury: a second randomised controlled trial. J Neurol Neurosurg Psychiatry 1998, 65: 177-183.

28. Corrigan JD, Smith-Knapp K, Granger CV: Outcomes in the first 5 years after traumatic brain injury. Arch Phys Med Rehabil 1998, 79:298-305.

29. Sunnerhagen KS, Johansson O, Herlitz J, Grimby G: Life after cardiac arrest; a retrospective study. Resuscitation 1996 3 I: | 35- 140.

30. Sander AM, Fuchs KL, High WM Jr., Hall KM, Kreutzer JS, Rosenthal $M$ : The Community Integration Questionnaire revisited: an assessment of factor structure and validity. Arch Phys Med Rehabil 1999, 80:1303-1308.

31. Cardol M, Beelen A, van den Bos GA, de Jong BA, de Groot IJ, de Haan RJ: Responsiveness of the Impact on Participation and Autonomy questionnaire. Arch Phys Med Rehabil 2002, 83:1524-1529.

32. Hoeymans $\mathrm{N}$, van Lindert $\mathrm{H}$, Westert GP: The health status of the Dutch population as assessed by the EQ-6D. Qual Life Res 2005, | 4:655-663.

33. Aaronson NK, Muller M, Cohen PD, Essink-Bot ML, Fekkes M, Sand erman R, Sprangers MA, te Velde A, Verrips E: Translation, validation, and norming of the Dutch language version of the SF36 Health Survey in community and chronic disease populations. J Clin Epidemiol 1998, 5 I:1055-1068.

34. von Steinbuechel N, Petersen C, Bullinger M: Assessment of health-related quality of life in persons after traumatic brain injury--development of the Qolibri, a specific measure. Acta Neurochir Suppl 2005, 93:43-49.

35. Broadbent DE, Cooper PF, FitzGerald P, Parkes KR: The Cognitive Failures Questionnaire (CFQ) and its correlates. Br J Clin Psychol 1982, 2 I (Pt I): I-I6.

36. Alderson AL, Novack TA: Reliable serial measurement of cognitive processes in rehabilitation: the Cognitive Log. Arch Phys Med Rehabil 2003, 84:668-672.

37. Vlaar AM, Wade DT: The Adult Memory and Information Processing Battery (AMIPB) test of information-processing speed: a study of its reliability and feasibility in patients with multiple sclerosis. Clin Rehabil 2003, I 7:386-393.

38. Sunderland A, Watts K, Baddeley AD, Harris JE: Subjective memory assessment and test performance in elderly adults. J Gerontol 1986, 41:376-384.

39. O'Donnell WE, Reynolds DM, De Soto CB: Neuropsychological impairment scale (NIS): initial validation study using trailmaking test (A \& B) and WAIS digit symbol (scaled score) in a mixed grouping of psychiatric, neurological, and normal patients. J Clin Psychol 1983, 39:746-748.

40. Vlaar AM, Wade DT: Verbal fluency assessment of patients with multiple sclerosis: test-retest and inter-observer reliability. Clin Rehabil 2003, I 7:756-764.

41. Spinhoven P, Ormel J, Sloekers PP, Kempen GI, Speckens AE, Van Hemert AM: A validation study of the Hospital Anxiety and
Depression Scale (HADS) in different groups of Dutch subjects. Psychol Med 1997, 27:363-370.

42. Sundin EC, Horowitz MJ: Impact of Event Scale: psychometric properties. Br / Psychiatry 2002, I 80:205-209.

43. van der Ploeg E, Mooren TT, Kleber RJ, van der Velden PG, Brom D: Construct validation of the Dutch version of the impact of event scale. Psychol Assess 2004, 16:16-26.

44. Bennett JA, Riegel B, Bittner V, Nichols J: Validity and reliability of the NYHA classes for measuring research outcomes in patients with cardiac disease. Heart Lung 2002, 3 I:262-270.

45. Kleinman L, Zodet MW, Hakim Z, Aledort J, Barker C, Chan K, Krupp L, Revicki D: Psychometric evaluation of the fatigue severity scale for use in chronic hepatitis C. Qual Life Res 2000 , 9:499-508.

46. de Haan R, Limburg M, Schuling J, Broeshart J, Jonkers L, van Zuylen $P$ : [Clinimetric evaluation of the Barthel Index, a measure of limitations in dailly activities]. Ned Tijdschr Geneeskd 1993, |37:9|7-92|

47. Turnbull JC, Kersten P, Habib M, McLellan L, Mullee MA, George S: Validation of the Frenchay Activities Index in a general population aged 16 years and older. Arch Phys Med Rehabil 2000, 8I: |034-1038.

48. Thornton M, Travis SS: Analysis of the reliability of the modified caregiver strain index. J Gerontol B Psychol Sci Soc Sci 2003, 58:SI27-32

49. Goossens ME, Rutten-van Molken MP, Vlaeyen JW, van der Linden SM: The cost diary: a method to measure direct and indirect costs in cost-effectiveness research. I Clin Epidemiol 2000, 53:688-695.

50. Oostenbrink JB, Bouwmans CAM, Koopmanschap MA, Rutten FFH: Handleiding voor Kostenonderzoek: Methoden en standaard kostprijzen voor economische evaluaties in de gezondheidszorg. Geactualiseerde versie edition. , College voor zorgverzekeringen; 2004.

5I. Ustun TB, Chatterji S, Bickenbach J, Kostanjsek N, Schneider M: The International Classification of Functioning, Disability and Health: a new tool for understanding disability and health. Disabil Rehabil 2003, 25:565-57I.

\section{Pre-publication history}

The pre-publication history for this paper can be accessed here:

\section{http://www.biomedcentral.com/1471-2261/7/26/prepub}

Publish with Bio Med Central and every scientist can read your work free of charge

"BioMed Central will be the most significant development for disseminating the results of biomedical research in our lifetime. "

Sir Paul Nurse, Cancer Research UK

Your research papers will be:

- available free of charge to the entire biomedical community

- peer reviewed and published immediately upon acceptance

- cited in PubMed and archived on PubMed Central

- yours - you keep the copyright 